culum. The absence of blood-vessels is characteristic of the tubercle. The lymphoid bodies are the first to appear at the point of attack of the bacillus tuberculosis. Their nucleus occupies nearly all their body; leaving only a small margin of protoplasm. On the sixth day after the inoculation of animals the epithelioid cells appear-the first distinctive step in the tubercular process. They appear at the point where the tuberculous bacilli are most numerous. Lastly the giant cells are formed, having their nuclei, ten to a hundred, arranged in the periphery of the cell. The epithelioid cells originate from the fixed epithelium and endothelium of the tissue involved. The giant cells are caused, most likely, by an inhibition of the normal cell divisions, the cell increasing in size notwithstanding. Between the cells and within them are grouped the bacilli. Twenty may be counted within a single section of a giant cell. The trabeculum is derived from the preëxisting connective tissue elements; changed, of course, by the new order of things. The roundness of the tubercle may be explained thus: In the beginning of the tubercle formation the cellgrowth is greater at the centre of the bacilli colony than at the periphery and, as there is a pressure exerted upon the centre by the periphery, a rounding up, as it were, results.

Regarding the uncertainty of the presence of the giant cells in tubercles we would say that their absence is comparatively very rare. If microörganisms are not necessary in every case to the development of giant cells, we know that the bacillus tuberculosis possesses this capability to a supreme degree. Yet, like many other things in nature, they may fail.

The tubercles may undergo: I, reformation; 2 , calcification; 3 , caseation; 4, liquefaction; 5 , suppuration; 6 , ulceration. Of these we will only speak of coagulative necrosis and suppuration. We cannot regard the caseous process (coagulative necrosis), as it occurs in the tubercle, in the sense used by Weigert in describing those processes tak ing place by reason of simple arrested circulation or nutrition ; as, for instance, in a non-infected infarct. We must look to the bacillus for a part of the change wrought. True, lack of nutrition has something to do with it, but the digestive power of the bacillus has more. And it may be thus looked upon as characteristic of the tubercular process, that no formation so small in size as a tubercle undergoes such a change.

Suppuration. - The bacillus tuberculosis never forms pus. Only when the so-called tubercular granulation tissue has become infected with the staphylococcus or streptococcus can true pus be formed. The contents of a purely tuberculous $a b-$ scess contains only a few round cells, but an amorphous shreddy material, the result of the digestive power of ths ptomaines from the bacillus tuberculosis upon the granulation tissue.
THE ETIOLOGY, PATHOLOGY AND TREATMEN'T OF ACUTE CATARRH OF THE UPPER AIR PASSAGES. Read before the American Rhinological Association, September

BY J. G. CARPEN'TER, M.D., OF STANFORD, KY.

The etiology of acute catarrh is either local or exciting, predisposing or constitutional: the use of tobacco, occupations attended with much dust, smoke, irritating gases, excessive moisture or dryness of the atmosphere, sudden changes of atmospheric temperature, as from a dry warm to damp or damp and cold weather, or from a warm room to the cold air without being sufficiently protected with wraps, getting the feet wet, standing or sitting for some time on a cold surface. A very pernicious habit is wetting the head, which is practiced daily by young ladies, and also by mothers and nurses, who wet the hair and scalp of children one or more times daily and thus develop acute or subacute catarrh, and the child is seldom over one attack before another begins; finally chronic catarrh of the upper air passages is established. Exposure to the night air without extra wraps is one of the most frequent causes of acute catarrh. Wearing damp clothes, changing beds and sleeping in rooms without fire (when not accustomed to the latter), exposure to currents of air, insufficient clothing, substituting light and thin goods for heavy and warm ones, changing boots for shoes or slippers, going without collar, cravat or cuffs, sitting up late at night and allowing the room to cool before retiring, facing the wind in traveling and not having the nose, mouth, throat and chest protected with extra wraps, removing the clothes and exposing the body in an almost nude state for half an hour or more before retiring, as many ladies do, are prevalent factors in the production of acute catarrh.

Mothers and nurses often develop acute inflammation of the upper air passages by getting up at night with children or patients in the cold air and not clothed properly; the body is not kept at the same temperature as when in bed, the consequence is that the integument is quickly chilled, its action suppressed, and through reflex irritation the mucous membrane of the upper air passages becomes congested or inflamed, as it is generally the weakest point, and most subject to irritation.

Too much clothing, as wearing sealskin sacques, chinchilla ulsters, fur collars and overshoes, on improper occasions as in pleasant weather, and not removing them on entering the house, church, theatre, or when making social calls, is equally as prone to develop acute catarrhal inflammation as insufficient clothing and undue exposure to inclement weather. The body should be clothed so as not to cause perspiration nor chilling."

I It is said that sealskin sacques have in a given time caused more deaths than small-pox in New York (Bosworth). 
Two barbarous customs are: first, the kind of hats or bonnets worn by women, which give no protection to the head; second, the manner in which boys and girls are dressed by the affluent, viz.: overclothing the trunk and head and allowing the limbs to be in an almost nude state, especially the knees, legs and feet, even in the coldest weather.

One of the most, if not the most prolific causes of catarrhal inflammation of the upper air passages is the treatment given infants immediately after birth. Generally the first thing that happens to the babe after the establishment of respiration is a cold in the head; or, as the nurse would say, "the little thing has the snuffles." It should be the exception and not the rule for infants to acquire a rhinitis shortly after birth from unnecessary exposure, chilling and sudden radiation of heat and evaporation of the body. It is quite common for the specialist to hear, "Doctor, my child has had a cold since its birth." The infant, before nor after severing the cord, should not be exposed, but wrapped in flannel of several layers in thickness. If very feeble, the cord should not be severed until pulsation has ceased, then artificial heat applied, the skin anointed with vaseline and the clothes quickly adjusted, an extra wrap applied and the babe placed at the mother's breast. If necessary, in the fall, winter or spring, artificial heat should be used. At the time of birth the temperature of the room, for the benefit of the babe, should range from $85^{\circ}$ to $100^{\circ} \mathrm{F}$. A sudden change of temperature from $20^{\circ}$ to $60^{\circ} \mathrm{F}$., from hot to cold, would injuriously affect a robust adult, nude or unprotected; how much greater must be the change and shock in the newly born babe, born and washed and dressed in a cold room and unnecessarily exposed. Many affections of the nose, throat, ears, eyes, trachea and bronchi could be avoided in children subsequent to birth if the accoucheur would use the proper precautions, and give the appropriate directions.

Constitutional causes of acute catarrh are rheumatism, malaria, measles, scarlatina, small-pox, diphtheria, uterine affections, especially endocervical metritis, neurasthenia. Excessive fatigue, either mental or physical, by impairing the general health, places the system in a condition favorable to acquiring catarrh of the upper air passages.

Pathology of Acute Catarrh.-The mucous membrane, the blood-vessels, nerves, $1 y$ mphatics of the rhino-pharyngeal cavities, supply or communicate with those of the eye, frontal, ethmoid and sphenoidal sinuses, the antrum, maxillars, the middle ear, lower pharynx, the tonsils, buccal cavity, larynx, trachea, bronchi and lungs ; consequently an irritation or inflammation of the superior respiratory tract is easily extended to these parts by reflex action or continuity of tissue.

The force of irritation and inflammation in acute catarrh of the upper air passages may be confined to the nares per se, the naso-pharyngeal chambers, or to the latter and the ethmoidal, sphenoidal and frontal sinuses and eyes, middle ears and antrum, or to the pharyngo-laryngeal cavities and trachea, and as the inflammation of one organ or region is of greater intensity and duration and predominates over the others, it is designated by that name, as rhinitis, rhino-pharyngitis, laryngitis, etc. Acute and subacute catarrh may end in resolution or in the chronic. When a chronic naso-pharyngitis is once established, it is quite easy to develop an otitis media, a conjunctivitis, a laryngitis, or rhinitis frontalis or ethmoiditis, sphenoiditis, or a tonsilitis. (The writer believes that catarrh of the upper air passages is the predisposing cause of phthisis in many cases-yes, in the majority of cases, it might be stated without exaggeration. Feebleness of constitution is inherited from consumptive parents, but consumption is not, it is acquired. "The discharge or secretion of catarrhal inflammation and the lymph channels afford the most favorable soil and habitat for the growth and reproduction of the bacillus tuberculosis," and when the catarrhal secretions contain bacilli they are capable of affecting healthy persons when dried, pulverized, and blown by the air and respired; hence, to avoid phthisis pulmonalis, keep the constitution normal, the upper respiratory passages healthy, and prevent a suitable soil for the development and reproduction of the bacillus tuberculosis. In every case of phthisis pulmonalis the writer has observed, rhino-pharyngeal catarrh, in one or more of its stages, was present.)

For convenience and perspicuity acute catarrh may be divided into three stages. I, the dry or congestive; 2 , the moist or liquefactive; 3 , the muco-purulent.

In the first stage there is an irritation of the mucous lining directly or reflexly; following this, there is dilatation of the blood-vessels with hyperæmia, redness, heat, tumefaction, and pain from pressure on the terminal ends of the nerves. Rhinoscopic examination shows a red or dark red, dry, congested, swollen appearance of the mucous lining, the blood-vessels are distended, enlarged, elongated and tortuous, and the lining often quite cedematous in those parts where it is loosely attached, or where it lies upon loose areolar tissue beneath.

Second Stage.-In addition to the first stage there are the following abnormal conditions, transudation of serum, diapodeses of the white corpuscles, infiltration of the connective tissue, cell proliferation, organization of $1 \mathrm{ymph}$. The walls of the mucous follicles are swollen, they are distended, abnormally active, and throw out an abundance of mucus upon the surface of the mucous membrane. The mucous and submucous areolar tissue are infiltrated, thickened and œdematous. On rhinoscopic examination the dry, red, 
glazed appearance has given way to a moist, juicy state of the surface; irregular elevations are seen corresponding to the distended crypts. There may also be infiltration and œdema of the turbinated processes and septum, causing nasal occlusion, enlargement and tenderness of the anterior cervical and maxillary glands, uvula, palate and tonsils.

The third stage is a continuation of the second, and is diagnosticated by a denudation or an erosion of the epithelial layer, leaving a raw surface, especially where there is much desquamation; supplementing this is a muco-purulent or a purulent secretion. Should resolution take place, the inflammatory products are absorbed, the bloodvessels contract to their normal size, excessive secretion is arrested, and the mucous surface presents its former healthy appearance.

On microscopic examination the mucous secretion contains tessellated and squamous epithelium, also columnar ciliated epithelium. The secretion from the upper pharynx is columnar ciliated epithelium; from the lower squamous epithelium; the epithelium of larynx below the superior vocal cords is columnar ciliated, above this point squamous; that of the trachea is also columnar ciliated. There are in addition, in the second state, mucus, white and red blood corpuscles, and pus corpuscles in the third stage.

Should acute catarrh not end in resolution, but in the chronic, there are developed in the mucous and submucous or connective tissue new layers of connective tissue elements. In the epithelial, there is an increased activity of cell growth by which it becomes abnormally thickened and hypertrophied, the glandular tissue is also involved, but to a limited extent. In the nasal cavities, the thickening is a regular and uniform hypertrophy, but appears irregular and nodular on the mucous surface. The hypertrophy is developed to its greatest extent in the turbinated bones; the inferior more than the middle, the latter more than the superior. The meatuses and septum are also affected by the infiltration.

Atrophic catarrh usually follows the hypertrophic and is generally late in developing, but may occur early, from recurrence of the acute or subacute catarrh, by which a deposition of lymph into the submucous connective tissue around the glands and follicles results, causing pressure on their orifices preventing the escape of mucus. After this pressure has existed for some time atrophy of the glandular and follicular tissue takes place.

\section{ABSCESS OF LIVER: RECOVERY.}

BY L. I. WILLIAMS, M.D.,

PASSED ASSISTANT-SURGEON, U. S. MARINE-HOSPITAL SERVICE.

C. B., seaman, aged 36 years, native of Maine, was admitted to the U. S. Marine Hospital, at
Boston, Mass., Feb. I4, I888, with symptoms of ulcerative colitis. He had had diarrhœa for two years, the discharges being watery and occasionally mixed with mucus and blood. There was tenderness on pressure over the entire course of the colon, notably the ascending portion. Examination of the rectum with the speculum gave a negative result. Patient's appetite was poor and his appearance anæmic. Ordered opii pulv. and bismuth subnitr., milk diet, and rest in bed. Under this treatment there was some improvement of the intestinal symptoms.

April 4th. Had a chill, followed by fever and sweating. Had been complaining of pain in right hypochondrium for several days previously. From this time patient had irregular chills and sweats, with increased temperature. Tenderness developed over the hepatic region, and the area of hepatic dulness was found to be abnormal in extent, reaching from just below the nipple to a point 3 centimetres below the border of the ribs. From the symptoms and history a probable diagnosis of abscess of the liver was made, and on April IIth the aspirator was introduced in the eighth intercostal space and $100 \mathrm{cc}$. of viscid, blood-stained pus evacuated.

April I 5th. $300 \mathrm{cc}$. of pus removed by the aspirator; pain relieved by the operation and patient slept better.

April 20th. $500 \mathrm{cc}$. of pus removed by the aspirator. As the abscess was evidently enlarging, it was determined to resort to a more radical operation.

On April 22d an incision was made in the eighth intercostal space, between the axillary and mammillary lines, and extending down to the surface of the liver. The chest cavity was obliterated at this point, owing to adhesion of the costal and diaphragmatic pleura. As no fluctuation could be detected, the incision was continued into the tissue of the gland, in the direction of the previous punctures, and the abscess cavity entered about 3 centimetres below the surface of the organ. The opening was enlarged by the forceps and rather more than $1000 \mathrm{cc}$, of reddish, grumous pus evacuated. The cavity was irrigated with $\mathrm{I}: 20,000$ warm corrosive sublimate solution, a large drainage tube introduced, a dresssing of absorbent cotton, wrung out of sublimate solution, applied and the whole covered by a large pad of oakum secured by a bandage. Cocaine was used as a local anæsthetic.

Next morning, April 23d, the dressings were found saturated with pus and bile, and patient was decidedly jaundiced. The abscess cavity was irrigated with solution of bichloride of mercury, I:20,000, but this caused so much pain and symptoms of collapse that it was never repeated. For the following notes I am indebted to AssistantSurgeon G. T. Vaughan, M. H. S., who at this time took charge of the case. 